\title{
Statements from International Union of Radio Science (URSI) on the Need for a Continuous Reference Timescale
}

\author{
Yasuhiro Koyama, Patrizia Tavella, and Judah Levine
}

\begin{abstract}
This article discusses the issues concerning the discontinuities in Coordinated Universal Time (UTC) that result from adding leap seconds to the UTC timescale. These issues have led to International Union of Radio Science (URSI) Resolution 1 on the need for a continuous timescale, and this resolution was approved at the URSI General Assembly and Scientific Symposium (GASS) 2021.
\end{abstract}

\section{Introduction}

Leap seconds are added to Coordinated Universal Time (UTC) on an irregular basis to ensure that the magnitude of the difference between UTC and Universal Time (UT1), a timescale on the basis of the rotation of Earth, is not greater than $0.9 \mathrm{~s}$. International Union of Radio Science (URSI) Commission A has often discussed the difficulties that are caused by this practice, and it raised this concern to the other URSI commissions. Commission A approved a resolution at General Assembly and Scientific Symposium (GASS) 2014 that recommended discontinuing the process of inserting leap seconds.

The leap second question is on the agenda for the meeting of the General Conference on Weights and Measures in 2022 and the World Radio Conference (WRC) of the International Telecommunication Union (ITU) in 2023, and the goal is to reach a final decision on this question at these meetings. URSI and other interested scientific institutions were officially invited to express opinions on the question.

To respond to this invitation, Commission A formed an ad hoc drafting group in February 2020 to draft a statement that would represent the opinion of URSI as a whole and not just Commission A. Commission A sent a notice to officials of all 10 commissions to inform them of this activity and to invite participation in the discussions. Twenty radio scientists joined the ad hoc group.

In the following section, we provide background information to explain the need for leap seconds and the

Manuscript received 28 December 2021.

Yasuhiro Koyama is with the National Institute of Information and Communications Technology, 4-2-1 Nukuikita, Koganei, Tokyo 184-8795, Japan; e-mail: koyama@nict.go.jp.

Patrizia Tavella is with the Bureau International des Poids et Mesures, Pavillon de Breteuil, 92312 Sèvres Cedex, France; e-mail: patrizia.tavella@bipm.org.

Judah Levine is with the National Institute of Standards and Technology, 325 Broadway, Boulder, Colorado 80305, USA; email: judah.levine@nist.gov. advantages and drawbacks of the current method of inserting them. Part of this work is based on [1] and on the discussion in the ad hoc drafting group.

\section{The Discontinuities of UTC}

Atomic clocks have been used to keep time and to define the length of the second since 1967. Previous definitions of time and time interval were based on astronomical observations linked to the rotation of Earth. These definitions were no longer adequate because the irregular variations in the rotation rate of Earth introduced corresponding variations in the definition of time interval and frequency and because the rotation rate is slowly decreasing, resulting in a corresponding increase in the length of the astronomical day and the length of the second derived from astronomical observations.

To support navigation in the 1970s, which was before the availability of global navigation satellite systems (GNSS), it was deemed necessary to maintain an agreement between UTC, which was widely disseminated and was realized by atomic clocks, and Universal Time (UT1), a timescale based on observations linked to the rotation of Earth. This agreement was needed so that civil time could be used as a proxy for the rotation angle of Earth, without the need for any correction. The timing experts working on atomic timescales and coordination of timekeeping all around the world met at the ITU-Radiocommunication Sector (ITU-R, which was the Consultative Committee on International Radio [CCIR] at the time) and defined a common code for time broadcasts. To keep UTC, defined by data from atomic clocks, in agreement with the Earth rotation, they chose to add a "leap second," whenever the magnitude of the difference between UTC and UT1 approaches $0.9 \mathrm{~s}$.

When a leap second is inserted, it is added as the last second of the day, usually on the last day of June or December. The official name of the leap second is 23:59:60, so that the last minute of a leap second day has $61 \mathrm{~s}$, numbered 0 to 60 . Thirty-seven leap seconds have been added to UTC from the beginning of this method in 1972 until the present (January 2022). The procedure for inserting leap seconds is described in the ITU-R Recommendation TF.460-6 (approved February 2002; https://www.itu.int/rec/R-REC-TF.460-6200202-I/en). The procedure also considers the possibility of "negative leap seconds," but these have never been used. 
A GNSS receiver determines its position by measuring the transit times of signals from several satellites that are observed simultaneously. The accuracy of this method depends on a smoothly varying satellite system timescale. It was considered operationally too difficult to insert a leap second into all satellites and receivers simultaneously, so GPS and most other navigation systems use a system time that was originally initialized to UTC but does not include leap seconds that were added after that instant. At the present time (January 2022), GPS time is ahead of UTC by $18 \mathrm{~s}$. The European Galileo system time has chosen the same labeling of seconds as the system time of GPS. Russian GLONASS, on the other hand, decided to follow UTC and add leap seconds, while the Chinese Beidou system time, based on the same considerations, synchronized the system time to UTC at the beginning of the operation and is now $4 \mathrm{~s}$ ahead of UTC. These navigation timescales are not an international standard, but they are very widely used because they are easily accessible with any common GNSS receiver. The differences in the system times can sometimes cause confusion, and it can be difficult to combine data from different constellations.

As we described previously, a leap second is inserted after second 23:59:59; it is labeled 23:59:60 and is followed by the second named 00:00:00 of the following day. However, digital clocks, which keep time internally as the number of seconds (and fractions) that have elapsed since some epoch, do not have the possibility to represent the second named 23:59:60. It is not practical to program digital devices to account for future leap seconds, because they are inserted on an irregular and unpredictable basis. Different solutions have been implemented to represent the time during the leap second. For example, some systems effectively stop the clock for an extra second and repeat the time corresponding to 23:59:59 a second time. Other systems stop the clock and repeat the time corresponding to 00:00:00 of the next day a second time. Other systems "smear" the leap second by a frequency adjustment, with some before the leap second, after the leap second, or both before and after. Google uses a frequency adjustment over the $24 \mathrm{~h}$ before the leap second, Facebook uses a frequency adjustment for $18 \mathrm{~h}$ after the leap second, and Alibaba uses a symmetrical smear from $12 \mathrm{~h}$ before to $12 \mathrm{~h}$ after the leap second. All of these solutions are not in agreement with UTC on the leap second day, many disagree with each other, and a common user cannot tell what method is used by a time source.

The problems with leap seconds and the many incompatible methods of realizing them have been discussed since early 2000 in the ITU, where the current leap second system was first recommended, in the metrological community of the Bureau International des Poids et Mesures (International Bureau of Weights and Measures; BIPM), and in several scientific organizations, such as URSI.
The ITU proposed to modify the leap second recommendation at the WRC in 2012 and 2015, but consensus was not reached, and a final decision was postponed. Resolution 655 of the WRC in 2015 asked to strengthen the cooperation between ITU-R and the BIPM, the International Committee for Weights and Measures (CIPM), the Conference General des Poids et Mesures (General Conference on Weights and Measures; CGPM), and other organization such as URSI. The organizations were encouraged to carry out a dialog and to submit a report at the next WRC in 2023.

There is a significant risk with the increasing number of widely available timing solutions, addressing the leap second question but not being compatible with UTC (or with each other): UTC, if not modernized, will become marginal and less universal; therefore, it will not be recognized and adopted as the international standard for timekeeping. These solutions have been designed and implemented by private third parties to satisfy internal requirements and have not been recognized or approved by the international community.

UTC, on the other hand, is computed by the BIPM with contributions from the timing laboratories of many countries and represents the work of hundreds of physicists and metrologists. It is widely recognized as the legal and practical source of time and frequency. The computation of UTC by the BIPM is under the authority of the CGPM, based on discussions among all member states and associates. The computation of UTC is not controlled by a single country, a single state department, foreign ministry, or any commercial entity. UTC is disseminated in each country by the legally recognized National Metrology Institute or timing authority. These institutions disseminate a real-time local approximation called UTC(k).

UTC is a worldwide time and frequency reference that must meet the needs of both current and future users. Many industrial and commercial applications depend on digital networks, and measurements of elapsed time require a timescale that is widely and easily available and is monotonic and smoothly varying. The different implementations of leap seconds and the easy availability of time signals from different global navigational satellites, which disagree with each other at the level of multiple seconds, are serious risks for the malfunctioning of the systems and seriously threaten the resilience of components of critical national infrastructure, such as telecommunications, energy transmission, and finance.

The BIPM and its Consultative Committee for Time and Frequency (CCTF) are working to obtain a consensus on the need for a continuous UTC, without leap seconds. A possible solution could be to extend the tolerance between UTC and UT1, the rotational time of the Earth, to $1 \mathrm{~min}, 1 \mathrm{~h}$, or to an unbounded limit. From the perspective of everyday timekeeping, the changes to and from daylight saving time have already weakened the connection between UTC and apparent solar time. However, the insertion of a leap second causes significant difficulties in the operation of computer 
networks and systems, telecommunication networks, and energy distribution.

There are still applications that depend on the UT1 timescale, and the value of UT1-UTC needs to be easily available to support these users. This information, with accuracy of the order of tens of microseconds, is currently available in Bulletin A of the International Earth Rotation and Reference Systems Service (https:// datacenter.iers.org/data/latestVersion/bulletinA.txt), and additional distribution methods would be very useful.

All members of the URSI Commission A drafting group, discussed previously, supported the importance of the UTC as a continuous timescale. Many members supported the idea of removing the current limit on the difference of UT1 and UTC, but there were also opposing opinions. One of the members was concerned that some organizations could not give an accurate and comprehensive evaluation of potential negative effects that would result from the elimination of leap second adjustments. This opinion was incorporated into the draft resolution by adding two conditions in the last sentence of the "resolves" part. The final version of the approved resolution is reported in the next section.

\section{The URSI Resolution 1 (GASS 2021)}

The URSI Council, considering that

a) the current practice of maintaining the Coordinated Universal Time (UTC) within $0.9 \mathrm{~s}$ of the Earth's rotation angle (UT1) by occasional leap second adjustments has been under discussion since the late 1990s;

b) the Radiocommunication Sector of the International Telecommunication Union (ITU-R) discussed the realization of timescales and dissemination of time signals via radiocommunication systems during its WRC 2015 and resolved in Resolution 655 to further and more widely study the various aspects of current and potential future reference timescales, including their impacts and applications, in cooperation with URSI and other relevant international organizations;

c) in 2018 the 26th General Conference on Weights and Measures (CGPM) formally confirmed in its Resolution 2 the definitions of International Atomic Time (TAI) and Coordinated Universal Time (UTC), and asked all relevant scientific unions and organizations to work together to develop a common understanding on reference timescales and their realization and dissemination, with a view to considering the present limitation on the maximum magnitude of UT1-UTC, so as to meet the needs of the current and future user communities; d) URSI Commission A organized a wider consultation with experts from various fields to request their opinions on the adoption of a continuous reference timescale,

noting that

a) the insertion of leap seconds has led to serious problems and breakdowns in contemporary applications, such as satellite navigation, distributed measurement systems, and computer networks, that require a continuous time reference.

b) In an attempt to minimize these problems, several actions have been put into practice by different users, either using a nonstandard continuous time reference (i.e., GPS time), or adopting different procedures to synchronize to UT1.

c) These actions have, in turn, caused confusion and errors for the users;

d) due to ambiguity during the insertion of a leap second, the metrological traceability to UTC that is required by some users is frequently not realized;

e) there are still concerns about unforeseeable effects caused by changing the current method of maintaining UTC to agree with UT1 within $0.9 \mathrm{~s}$;

f) UT1 is necessary for applications in the space industry, Earth-based observations, for transformation between fixed and rotating reference systems, and for these applications real-time UT1 signal dissemination is needed;

g) the definitive values of UT1-UTC are provided by the International Earth Rotation and Reference Systems Service (IERS) on the Internet and are also available via other time dissemination techniques by radio signals, global navigation satellite systems, and Internet time protocols;

h) TAI should not be considered as an option to achieve a continuous reference timescale since in its present form, it provides only a frequency reference and is not disseminated by clocks,

recognizing that

a) URSI passed the URSI resolution of strengthening the URSI and ITU relationship in its General Assembly in Lille, 1996, and resolved that the board shall work with ITU in the identification of topic areas of mutual concern and prepare URSI statements on such topics in an appropriate form;

b) an URSI-wide working group was formed in 2002, and the risks that the occasional leapsecond adjustments might cause were identified; 
c) Commission A (electromagnetic metrology) of URSI expressed its opinion in 1999 that the procedure of leap-second insertions should be stopped, and thus UTC should become a continuous reference timescale and that this position was confirmed in 2014 by a resolution of Commission A,

resolves for URSI to make the following statements:

1) All global navigation satellite systems are requested to consider broadcasting UT1- UTC to a precision of a millisecond or better, within the constraints of their available funding and development latencies. In addition, systems providing UT1-UTC over the Internet should be hardened against cyberattacks and should be supplemented with additional secondary sources for users who only require annual knowledge of UT1- UTC.

2) There are various risks caused by the adjustment of leap seconds on UTC that are not predictable over the long term.

3) A unique and continuous reference timescale is essential for scientific research and related activities in Radio Science.

4) Many of the technological concerns associated with adapting systems and software can be solved and that challenge can be justified in comparison to the scientific and operational benefits of a continuous reference timescale.

5) Therefore, the present limitation on the maximum magnitude of UT1-UTC should be withdrawn after a suitable period of public notice provided that real-time UT1-UTC dissemination is achieved and that no currently unforeseen problem is identified before 2023.

\section{Conclusion}

The debate on the need of a continuous reference timescale has been going on for more than 20 years, and the time for a decision has come. URSI has comprehensively evaluated the impact of this decision in radio science activities and hopes, with the 2021 GASS Resolution, to give a valuable contribution to the international debate and to support the way forward toward a continuous UTC.

\section{Acknowledgments}

The authors appreciate the cooperation of Sarang Kulkarni, Rich Bradley, Jacob Halevy-Politch, Joseph Achkar, Liu Min, Felicitas Arias, Francesco Lamonaca, Fang Fang, Demetrios Matsakis, Subramaniam Ananthakrishnan, Amitava Sen Gupta, Steven Weiss, Bhal Chandra Joshi, Sayyad Shafiyoddin Badruddin, J. Mauricio Lopez R. Tianchu Li, and Carsten Rieck, who volunteered to join the ad hoc drafting group, for helpful contributions.

\section{Reference}

1. J. Levine and P. Tavella, "Discontinuities in Coordinated Universal Time: Advantages and Perspectives," International Union of Radio Science General Assembly and Scientific Symposium (URSI GASS 2021), Rome, Italy, August 28-September 4, 2021, Mo-A01-AM2-2. 